\title{
Strongly Nonlinear Transverse Perturbations in Phononic Crystals
}

\author{
S. Nikitenkova ${ }^{1}$ and E. Pelinovsky ${ }^{2,3}$ \\ ${ }^{1}$ Radiophysics Department, Lobachevsky State University of Nizhny Novgorod, Nizhny Novgorod 603950, Russia \\ ${ }^{2}$ Department of Nonlinear Geophysical Processes, Institute of Applied Physics, Nizhny Novgorod 603950, Russia \\ ${ }^{3}$ Department of Applied Mathematics, Nizhny Novgorod Technical University, Nizhny Novgorod 603950, Russia
}

Correspondence should be addressed to S. Nikitenkova; snikitenkova@gmail.com

Received 7 March 2014; Accepted 3 June 2014; Published 2 July 2014

Academic Editor: Gani Stamov

Copyright (C) 2014 S. Nikitenkova and E. Pelinovsky. This is an open access article distributed under the Creative Commons Attribution License, which permits unrestricted use, distribution, and reproduction in any medium, provided the original work is properly cited.

\begin{abstract}
The dynamics of the surface heterogeneities formation in low-dimensional phononic crystals is studied. It is shown that phononic transverse perturbations in this medium are highly nonlinear. They can be described with the help of the Riemann wave and may form stable wave structures of the finite amplitude. The Riemann wave deformation is described analytically. The Riemann wave time existence up to the beginning of the gradient catastrophe is calculated.
\end{abstract}

\section{Introduction}

Interest to low-dimensional structures is caused by the unique properties of these materials, allowing using them in many areas and, first of all, in nanoelectronics including active components of nanomechanical systems (NEMS) [1]. The key role of phonon processes in low-dimensional structures is well known $[2,3]$. Transverse thermal vibrations of atoms in low-dimensional structures in the direction perpendicular to the plane structure can lead to the formation of the complicated surface elevation, increase the effective structure thickness, and affect its physical properties. For example, in the transverse thermal vibrations of graphene atom may reach relatively high amplitudes even in normal conditions that is approximately an interatomic distance $[4,5]$. The additional structure strain often used in NEMS complicates the transverse vibrations dynamics of the lattice even more [6].

Therefore, phononic processes in low-dimensional structures have a complicated sometimes even a strongly nonlinear character. Strong nonlinearity can lead to the formation of the stable wave structures with a wide frequency spectrum. The understanding of nonlinear features of the similar processes even in the frames of some simplest models may presume definite interest from the point of view of defining the adequate restrictions to the application of low-dimensional materials.

The process of the surface heterogeneity formation as a result of nonlinearity of oscillations of the phononic crystal lattice is considered in the paper. Nonlinear waves are studied in the frames of the conceptual model of the atom chains connected with the elastic bonds; the mentioned model is described in Section 2. The nonlinearity of the model is not limited. This model, though simplified from the point of view of the physical processes in the phononic crystals, allows identifying many features of the nonlinear behavior of phononic crystals. The nonlinear Riemann travelling wave is studied within the frame of this system in Section 3. The results of the conditions of the travelling wave existence up to the onset of the gradient catastrophe are obtained. The definite example of the transformation of the initially sinusoidal disturbance is analyzed in Section 4 . The results obtained are summarized in Conclusion.

\section{The Discrete Model of the Transverse Oscillations}

A typical phononic crystal can be regarded as a spatially regulated structure whose discreteness must be considered when 


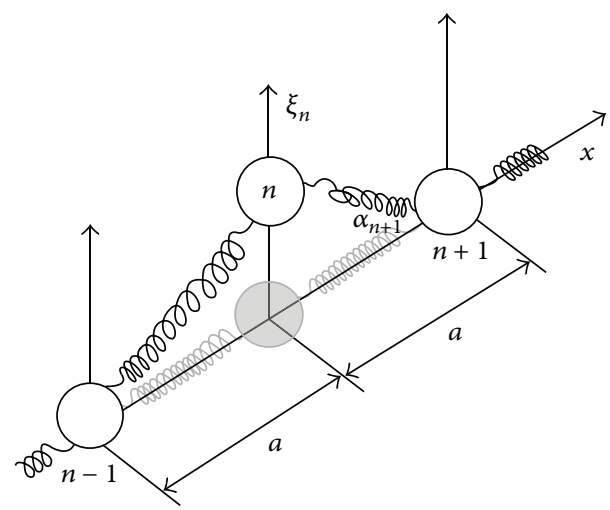

FIGURE 1: Transverse oscillations of equal mass particles in an atomic chain.

studying the dynamics of phonons. Discrete mathematical models are an effective tool for theoretical and experimental studies of the behavior of low-dimensional structures. The basic discrete model for the study of transverse oscillations is a chain of atoms the movement of which is limited by guides in the form of parallel straight lines lying on one plane at equal distance from each other (Figure 1) [7, 8].

The equation of motion for the atom with number $n$ can be written as

$$
m \frac{d^{2} \xi_{n}}{d t^{2}}=F_{n-1}+F_{n+1}
$$

where $m$ is the mass, $\xi_{n}$ is the deviation of the mass along the guideline from equilibrium position $\xi_{n}=0$, and $F_{n \pm 1}$ is transverse components of the forces acting from the neighboring masses. The forces acting on the atoms in real phonon crystals are determined by the interatomic interaction potential. In the case of small perturbations of the chain, the harmonic approximation of the interaction potential can be used, and the forces can be calculated by Hooke's law [9]:

$$
F_{n \pm 1}=-K \Delta l_{n \pm 1} \sin \left(\alpha_{n \pm 1}\right)
$$

where $K$ is the analogue of Hook's constant, $\Delta l_{n+1}$ is the extension of the spring between the masses with $n$ and $(n \pm 1)$, and $\alpha_{n \pm 1}$ is the local angle of the deviation from the chain on axis. The extension $\Delta l_{n+1}$ of the spring during the motion of the masses can be calculated by a simple formula:

$$
\Delta l_{n+1}=\left(\Delta l_{0}+\sqrt{\left(\xi_{n+1}-\xi_{n}\right)^{2}+a^{2}}-a\right)
$$

where $\Delta l_{0}$ is the extension in the equilibrium position related to the preliminary tension of the spring. The value $\sin \left(\alpha_{n \pm 1}\right)$ can be found from the geometric relations:

$$
\sin \left(\alpha_{n+1}\right)=\frac{\left(\xi_{n+1}-\xi_{n}\right)}{\sqrt{\left(\xi_{n+1}-\xi_{n}\right)^{2}+a^{2}}} .
$$

The extension $\Delta l_{n-1}$ and the angle $\alpha_{n-1}$ are calculated in the analogous way. As a result, we get

$$
F_{n+1}=-K\left(\xi_{n+1}-\xi_{n}\right) \frac{\Delta l_{0}+\sqrt{\left(\xi_{n+1}-\xi_{n}\right)^{2}+a^{2}}-a}{\sqrt{\left(\xi_{n+1}-\xi_{n}\right)^{2}+a^{2}}} .
$$

Let us consider the small vibrations (as it will be shown below, this does not mean that the oscillations are weakly nonlinear), using the approximation $\left|\xi_{n}-\xi_{n \pm 1}\right| \ll a$ and $\Delta l_{0} \ll a$.

The nonlinearity introduced in this manner has a geometrical character (see also [10]) and is not dependent on the physical properties of the phonon crystal.

Then (1) can be rewritten in the following form:

$$
\begin{aligned}
m \frac{d^{2} \xi_{n}}{d t^{2}}= & -K \frac{\Delta l_{0}}{a}\left(2 \xi_{n}-\xi_{n+1}-\xi_{n-1}\right) \\
& -\frac{K}{2 a}\left[\left(\xi_{n}-\xi_{n-1}\right)^{3}-\left(\xi_{n+1}-\xi_{n}\right)^{3}\right]
\end{aligned}
$$

The elastic continuum model provides an adequate description of acoustic phonons in nanostructures [2, 9]. To describe the transverse perturbations let us pass over to the continuum limit, assuming the wavelength is large compared with the spatial period $a$ of the chain. The discrete coordinate $n a$, determining the position of atoms in the chain, is replaced by a continuous coordinate $x$.

Let us direct the axis $x$, along which the wave propagates, perpendicular to the guideline (see Figure 1). We will consider

$$
\xi_{n}=\xi(x), \quad \xi_{n+1}=\xi(x+a), \quad \xi_{n-1}=\xi(x-a)
$$

and expand variables in a small period $a$. In the longwave limit, the dispersion law of acoustic phonons is well approximated by a linear dependence [9], so the expansion will retain only the first two terms:

$$
\left(\xi_{n}-\xi_{n-1}\right)^{3}-\left(\xi_{n+1}-\xi_{n}\right)^{3} \approx-3 a^{4}\left(\frac{\partial \xi}{\partial x}\right)^{2} \frac{\partial^{2} \xi}{\partial x^{2}} .
$$

As a result, we obtain the nonlinear wave equation (herein after we will omit number $n$ ):

$$
\frac{\partial^{2} \xi}{\partial t^{2}}-c^{2} \frac{\partial^{2} \xi}{\partial x^{2}}=\beta\left(\frac{\partial \xi}{\partial x}\right)^{2} \frac{\partial^{2} \xi}{\partial x^{2}}
$$

where the nonlinear coefficient and the square of the sound speed are given by the following expressions:

$$
\beta=\left(\frac{3 K}{2 m}\right) a^{2}, \quad c^{2}=\left(\frac{K}{m}\right) \Delta l_{0} a .
$$

Let us perform the transformation of the variables for the nonlinear wave equation (9) not to contain constants:

$$
\begin{aligned}
& \frac{\partial^{2} z}{\partial \tau^{2}}-\frac{\partial^{2} z}{\partial x^{2}}=\left(\frac{\partial z}{\partial x}\right)^{2} \frac{\partial^{2} z}{\partial x^{2}} \\
& z=\sqrt{\frac{2 \Delta l_{0}}{3 a}} \xi, \quad \tau=\sqrt{\frac{K \Delta l_{0} a}{m} t .}
\end{aligned}
$$


The lattice oscillations in the framework of (9) can be described in two ways-in the form of standing and traveling waves. The solutions of (9) in the form of standing waves of small amplitudes in a bounded system were obtained in $[8,11]$, strongly nonlinear vibrations at large amplitudes in [12]. However, the undoubted practical interest presumes the transfer of energy in a rather long phononic crystal lattice model, which should be described by the solutions of (9) in the form of traveling waves.

\section{Dynamics of Deformation in the Approximation of Riemann Waves}

In finding the solution, (11) will not assume linearity low. To find solutions it is convenient to move from a nonlinear wave equation (11) to a system of quasilinear equations using substitutions:

$$
\begin{aligned}
& \frac{\partial z}{\partial x}=u \\
& \frac{\partial z}{\partial \tau}=v
\end{aligned}
$$

Substituting (12) into (11), we obtain the nonlinear first-order equation for two unknown functions:

$$
\frac{\partial v}{\partial \tau}-\frac{\partial u}{\partial x}=u^{2} \frac{\partial u}{\partial x}
$$

The second missing equation is obtained by crossdifferentiation (12)

$$
\frac{\partial u}{\partial \tau}=\frac{\partial v}{\partial x}
$$

The system of (13) and (14) is hyperbolic and, therefore, admits solutions in the form of propagating waves. In each wave, even nonlinear, the variables are linked, so we assume that $v=v(u)$. Then, the system of (13) and (14) is reduced to

$$
\begin{gathered}
\frac{\partial u}{\partial \tau}-\frac{\partial v}{\partial u} \frac{\partial u}{\partial x}=0, \\
\frac{\partial v}{\partial u} \frac{\partial u}{\partial \tau}-\left(1+u^{2}\right) \frac{\partial u}{\partial x}=0 .
\end{gathered}
$$

System (15) is a system of linear equations with respect to the derivatives $\partial u / \partial \tau$ and $\partial u / \partial x$. For the existence of a nontrivial solution it is essential and enough that its determinant be zero, which leads to the determination of the required connection between the functions $v=v(u)$ :

$$
\left(\frac{d v}{d u}\right)^{2}=1+u^{2}
$$

Extracting only one root in (16), corresponding to the wave traveling to the right, we obtain the equation:

$$
\frac{d v}{d u}=\sqrt{1+u^{2}}
$$

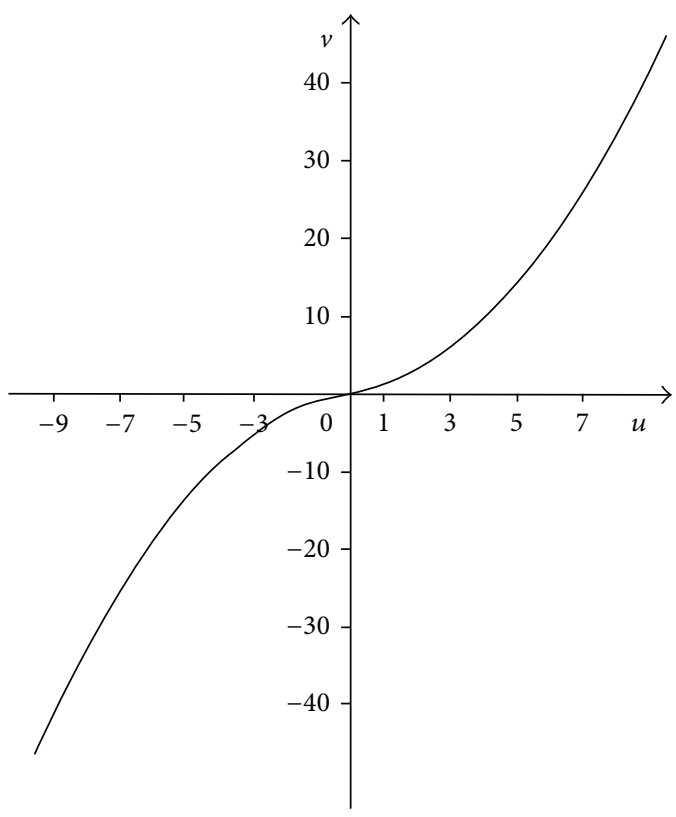

Figure 2: Dependence $v=v(u)$ in (18).

Equation (17) is easily integrated into

$$
v=\int \sqrt{1+u^{2}} d u=\frac{u \sqrt{1+u^{2}}}{2}+\frac{\operatorname{Arsh}(u)}{2}+C,
$$

where $C$ is the arbitrary constant of the integration. From the condition that at infinity $u \rightarrow 0$ and $v \rightarrow 0$ the value is $C=0$. This curve is displayed in Figure 2.

In a traveling wave due to (18), the equations of system (15) become identical; therefore, later either of them can be used. It is conveniently rewritten as

$$
\frac{\partial u}{\partial \tau}+U(u) \frac{\partial u}{\partial x}=0
$$

where

$$
U(u)=\sqrt{1+u^{2}} .
$$

The solution of (19), satisfying the initial condition

$$
\begin{gathered}
z(x, \tau=0)=F(x), \\
u(x, \tau=0)=\frac{d F(x)}{d x}=f(x),
\end{gathered}
$$

is expressed in the form of a simple wave or the Riemann wave:

$$
u=f\lfloor x-U(u) \tau\rfloor,
$$

Formation of the Riemann waves in monatomic chain with longitudinal displacement and first-neighbors interactions is discussed in detail in the book [9]. The Riemann waves in monatomic chain with transverse displacement have not been studied previously. However, some experimental 
data, given in works $[13,14]$, can be considered an indirect evidence of their existence.

To calculate the wave profile $z(x, \tau)$ let us move to the implicit Riemann variable:

$$
y=x-U(u) \tau=x-\sqrt{1+u^{2}} \tau
$$

Then, the spatial derivative becomes the function of the new variable $u=f(y)$ and according to (12), the perturbation can be found:

$$
z(x, \tau)=\int u(x, \tau) d x=\int u(y) \frac{d x}{d y} d y .
$$

From (23) it follows that

$$
d y=\left[1-\tau \frac{\partial U(u)}{\partial u} \frac{\partial u}{\partial x}\right] d x
$$

The derivative $\partial u / \partial x$ entering (25) can be calculated as

$$
\frac{\partial u}{\partial x}=\frac{\partial u}{\partial y} \frac{\partial y}{\partial x}=\frac{\partial u}{\partial y}\left[1-\tau \frac{\partial U(u)}{\partial u} \frac{\partial u}{\partial x}\right]
$$

Then

$$
\frac{\partial u}{\partial x}=\frac{\partial u / \partial y}{1+\tau(\partial U(u) / \partial u)(\partial u / \partial y)}
$$

and the integral (24) is converted to

$$
z(x, \tau)=\int u(y) d y+\tau \int u \frac{d U(u)}{d u} d u .
$$

The value of the second integral in (28) can be found by using the explicit expression for $U(u)$, given by formula (23):

$$
\int u \frac{d U(u)}{d u} d u=\int \frac{u^{2}}{\sqrt{1+u^{2}}} d u=\frac{u \sqrt{1+u^{2}}}{2}-\frac{\operatorname{Arsh}(u)}{2}
$$

and the arbitrary constant of the integration was obtained due to the assumption that if $u \rightarrow 0$, the value of the integral must also tend to zero.

As $u(x, \tau)=\partial z(x, \tau) / \partial x$ the value of the first integral in (28) is equal to

$$
\int u(y) d y=F(y)
$$

Then we finally obtain the expression for the displacement

$$
\begin{aligned}
z(x, \tau)= & F\left(x-\sqrt{1+f(y)^{2}} \tau\right) \\
& +\left[\frac{f(y) \sqrt{1+f(y)^{2}}}{2}-\frac{\operatorname{Arsh}(f(y))}{2}\right] \tau,
\end{aligned}
$$

where $y$ is represented by formula (23). Formulas (22), (23), and (31) describe a propagating nonlinear deformable wave in the phononic crystal. The concrete example of the deformation of the original disturbance on the example of the harmonic impulse is considered in Section 4.

The nonlinear deformation of the wave leads to the formation of a steep front and subsequent ambiguity of the solution. This process, called a gradient catastrophe, is well known in nonlinear mathematical physics $[15,16]$. Let us define here the onset of the gradient catastrophe. It is sufficient to use expression (26) for the spatial derivative of the function $u(x, \tau)$, which is conveniently rewritten as

$$
\begin{aligned}
\frac{\partial u}{\partial x} & =\frac{d f(y) / d y}{\left[1+(d f(y) / d y)\left(f(y) / \sqrt{1+f(y)^{2}}\right) \tau\right]} \\
& =\frac{d f(y) / d y}{\left[1+(d / d y) \sqrt{1+f(y)^{2}} \tau\right]}
\end{aligned}
$$

where $y$ again is defined by formula (23). As we can see, the derivative increases in those sectors of the wave where $d f / d y<0$ and it becomes infinite during the time

$$
\begin{aligned}
T_{\mathrm{br}} & =\frac{1}{\operatorname{Max}_{x}\left[-(d / d x) \sqrt{1+f(x)^{2}}\right]} \\
& =\frac{1}{\operatorname{Max}_{x}\left[-(d / d x) \sqrt{1+((d / d x) F(x))^{2}}\right]} .
\end{aligned}
$$

The "breaking" of the front of the wave occurs at the point where the coordinate $X_{\mathrm{br}}$ satisfies the following conditions:

$$
\left.\left(\sqrt{1+f(x)^{2}}\right)^{\prime \prime}\right|_{x=X_{\mathrm{br}}}=0,\left.\quad\left(\sqrt{1+f(x)^{2}}\right)^{\prime \prime \prime}\right|_{x=X_{\mathrm{br}}}<0 .
$$

From the first equation (33) it follows that the breaking coordinate $X_{\mathrm{br}}$ is the solution of the equation:

$$
\left(f(x)^{\prime}\right)^{2}+f(x)\left(1+f(x)^{2}\right) f(x)^{\prime \prime}=0 .
$$

Using (32) and (34) the breaking time can be calculated as

$$
T_{\mathrm{br}}=\left.\frac{1}{\sqrt{-f(x)^{3} f(x)^{\prime \prime}}}\right|_{x=X_{\mathrm{br}}} .
$$

In the transition to the physical time it is

$$
\widetilde{T}_{\mathrm{br}}=\sqrt{\frac{m}{k \Delta l_{0} a}} T_{\mathrm{br}}
$$

Thus, the wave breaking time, which can be interpreted as the accumulation time of nonlinear effects in the environment, depends not only on the profile of the initial disturbance, but also on the parameters of the medium and the initial mass chain tension. 
In the case of small perturbations (the weakly nonlinear Riemann wave) the approximate formula follows from (31):

$$
\begin{gathered}
z(x, \tau)=F\left(x-\left(1+[f(y)]^{2}\right) \tau\right), \\
y=x-\left(1+[f(y)]^{2}\right) \tau
\end{gathered}
$$

and in the case of strongly nonlinear Riemann wave

$$
\begin{array}{r}
z(x, \tau)=F(x-|f(y)| \tau)+\frac{f(y)|f(y)|}{2} \tau, \\
y=x-|f(y)| \tau .
\end{array}
$$

\section{The Propagation of Harmonic Perturbation}

Surface structuring in a phononic crystal can be considered on the basis of the model of the temporal wave evolution in a chain of masses, perturbed at the initial moment following the harmonic law:

$$
F(x)=A \sin (k x)
$$

with the constant amplitude $A$ and the wave number $k$. Let the wave with the initial profile get distributed in the positive axis $x$ direction. Then the Riemann wave for the given initial perturbation is of the following form:

$$
u=A k \cos \left\lfloor x-\sqrt{1+u^{2}} \tau\right\rfloor .
$$

Following the conditions from (34), we find that the wave breaking will occur in two points of the profile and this pattern will be repeated across the length of the wave

$$
\begin{aligned}
X_{\mathrm{br}} & =\frac{1}{k} \arccos \left[ \pm \frac{\sqrt{\sqrt{1+(A k)^{2}}-1}}{A k}\right] \\
& =\frac{1}{k} \arcsin \left( \pm \frac{\sqrt{1+A^{2} k^{2}-\sqrt{1+A^{2} k^{2}}}}{A k}\right) .
\end{aligned}
$$

Formula (42) allows us to estimate the position of the "breaking" point depending on its amplitude. When the amplitude tends to zero, this formula (42) can be approximated by the following expression:

$$
X_{\mathrm{br}} \approx \pm \frac{1}{k} \operatorname{arc} \cos \left(\frac{\sqrt{2}}{2}\right)= \pm \frac{\pi}{4 k}= \pm \frac{\lambda}{8},
$$

where $\lambda=2 \pi / k$ is the wavelength. At large amplitudes formula (42) may be replaced by

$$
X_{\mathrm{br}} \approx \pm \frac{1}{k} \operatorname{arc} \cos (0)= \pm \frac{\pi}{2 k}= \pm \frac{\lambda}{4}
$$

Thus, the position of the point with the unlimited slope is relatively weakly dependent on the wave amplitude, and it varies from $\lambda / 8$ to $\lambda / 4$.

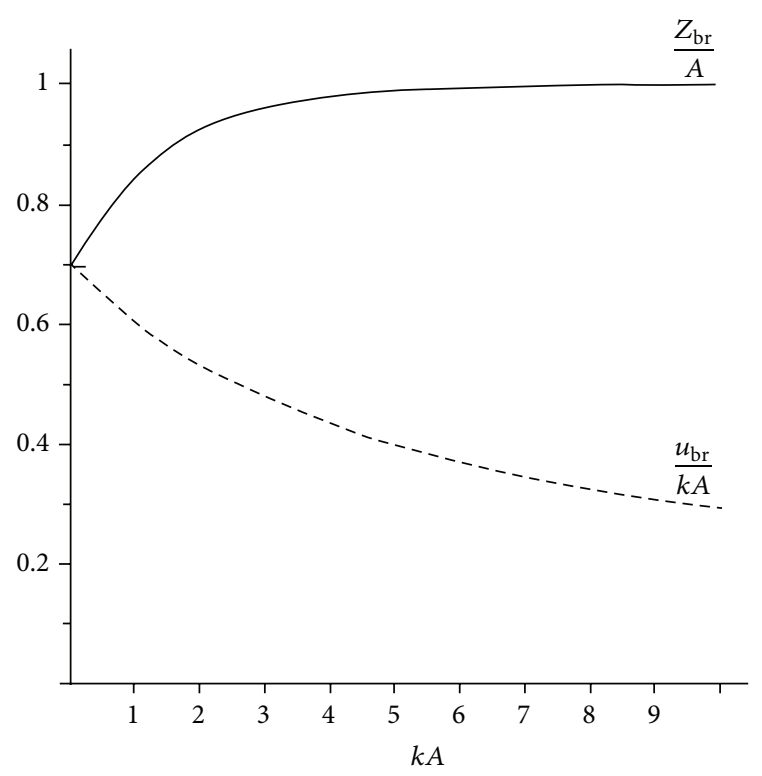

FIGURE 3: Dependence of the normalized breaking values $Z_{\mathrm{br}}$ and $u_{\mathrm{br}}$ on the value $k A$.

The "breaking" time according to formula (36) can be calculated explicitly:

$$
T_{\mathrm{br}}=\frac{1}{A^{2} k^{3} \cos \left(k X_{\mathrm{br}}\right)^{2}}
$$

or

$$
k T_{\mathrm{br}}=\frac{1}{\sqrt{1+A^{2} k^{2}}-1} .
$$

Accordingly (46) the wave of a small amplitude "breaks" for a very long time, and with the increasing amplitude the onset of the gradient catastrophe decreases rapidly.

The corresponding value of the breaking point $u(x, \tau)$ can be found from (40):

$$
u_{\mathrm{br}}=\sqrt{\sqrt{1+A^{2} k^{2}}-1}
$$

Then the value of the function $z(x, \tau)$ at the moment of breaking can be calculated by formula (31) provided by (41), (42), and (47):

$$
\begin{aligned}
& z_{\mathrm{br}}= \frac{\sqrt{1+A^{2} k^{2}-\sqrt{1+A^{2} k^{2}}}}{k} \\
&+\frac{1}{2}\left[\left(\left(1+A^{2} k^{2}\right)^{1 / 4} \sqrt{\sqrt{1+A^{2} k^{2}}-1}\right.\right. \\
&\left.-\operatorname{Arsh}\left(\sqrt{\sqrt{1+A^{2} k^{2}}-1}\right)\right) \\
&\left.\times\left(\sqrt{\sqrt{1+A^{2} k^{2}}-1}\right)^{-1}\right] .
\end{aligned}
$$




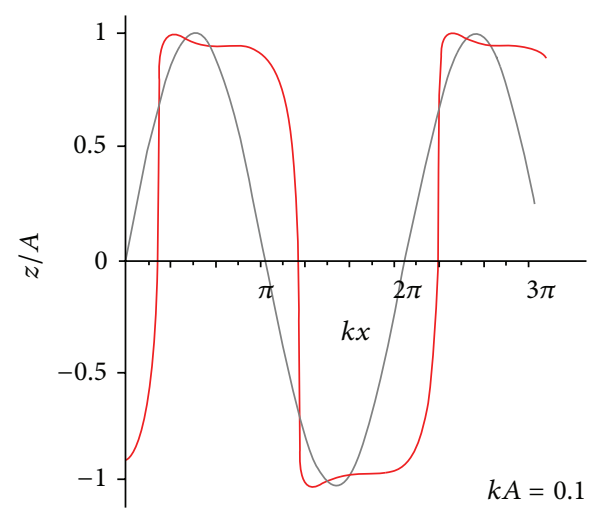

(a)

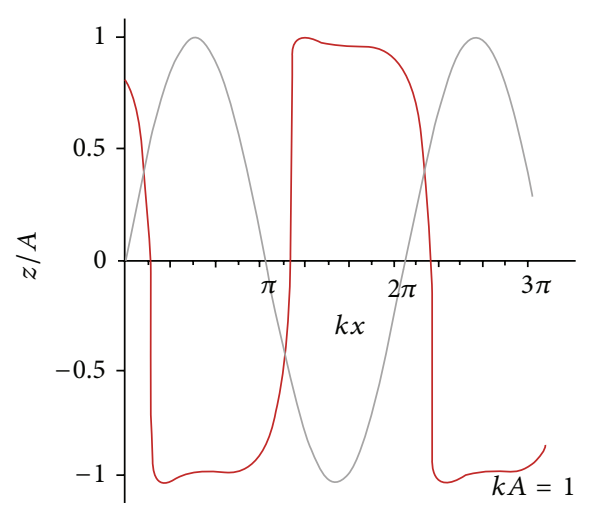

(c)

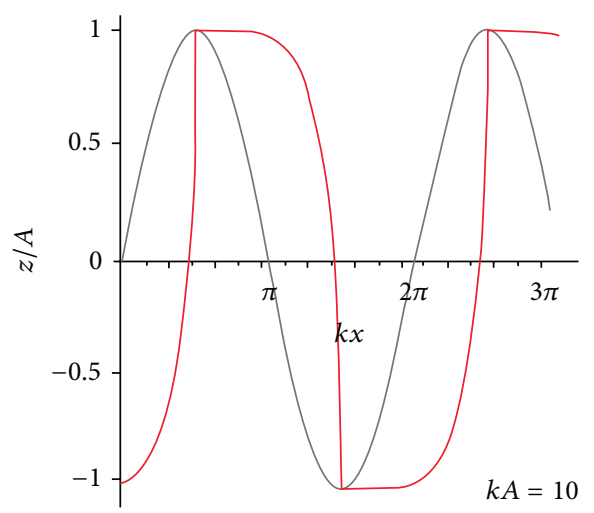

(e)

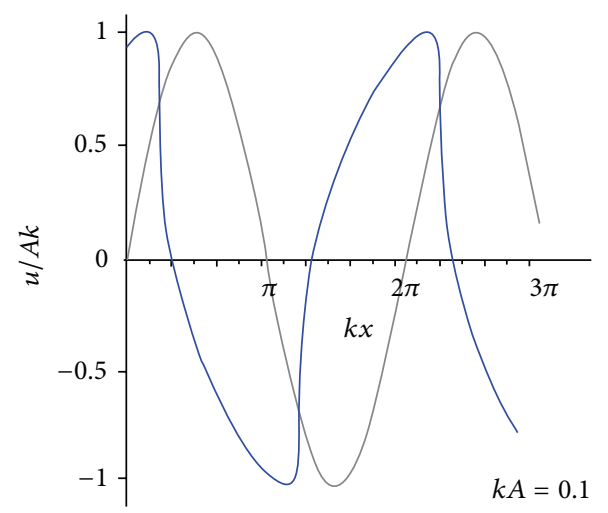

(b)

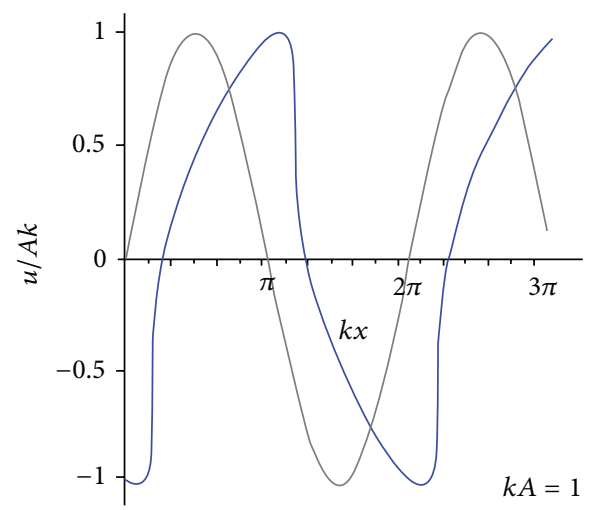

(d)

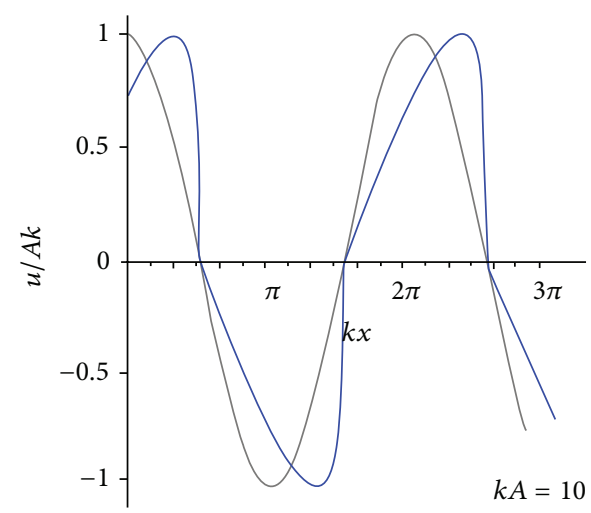

(f)

FIGURE 4: Shapes of the $z(k A) / A$ and $u(k A) / A k$ at the initial time and the breaking time: (a)-(b) $k T_{\text {br }}=200.5$ and $k A=0.1$; (c)-(d) $k T_{\text {br }}=2.41$ and $k A=1$; (e)-(f) $k T_{\text {br }}=0.11$ and $k A=10$.

The plot of points of breaking $z_{\mathrm{br}}$ and $u_{\mathrm{br}}$, normalized to the value of the amplitude and value of the amplitude multiplied wave number $k$, respectively, depending on the values of the quantity $A k$ is shown in Figure 3.

Temporal evolution of the transverse perturbations $z(x, \tau)$, normalized to the amplitude of the initial perturbation, calculated by formulas (18), (20), (22), and (31) for the harmonic initial perturbation with the values $A k=0.1$, $A k=1$, and $A k=10$, is represented in Figure 4.

From Figure 4 it is seen that with the propagation of the harmonic perturbation the wave profile becomes asymmetric and the asymmetry for the functions $z(x)$ and $u(x)$ is shown differently. With the time and the increase of the propagated distance, the steepening of the wave front $u(x)$ takes place. Initially sinusoidal wave profile $z(x)$ is transformed into a trapezoidal one. This agrees well with the results of the wave transformation in a cubic nonlinear medium obtained in $[17,18]$. The transformation of the profile appears faster and is more pronounced for large-amplitude waves.

It is worth mentioning that at the moment of the onset of the gradient catastrophe in the profile $u(x)$ the singularity of type $x^{1 / 3}$ is formed, as it was recently shown for hyperbolic 
equations of a relatively general form $[18,19]$. Since the function $z(x)$ is the integral of $u(x)$, then at the breaking point the singularity is very weak $\left(x^{4 / 3}\right)$ and that is why it is not visible in Figures 4(a), 4(c), and 4(e) in contrast to the graphs in Figures 4(b), 4(d), and 4(f).

\section{Conclusion}

The considered discrete model of the phononic crystal helps to reveal certain consistent patterns of energy transfer of thermal motion in low-dimensional phononic crystals. Collective displacements of transverse phonons in a longphononic crystal lead to the formation of traveling waves. As a result of the nonlinearity of the transverse lattice oscillations stable wave structures of the finite amplitude can be formed on the surface of the phononic crystal. In the long-wave approximation, the nature of transverse deformation of the lattice of the phononic crystal can be described by Riemann waves. The analytical expression of such Riemann wave allows establishing the dependence of the transverse deformation of the phononic crystal on its parameters allowing us to control the formation of surface structures. The time existence of the Riemann is determined. This time can be interpreted as the characteristic time of the accumulation of nonlinear effects that should be taken into consideration when determining the appropriate limitations on the use of low-dimensional materials. The stronger appearance of nonlinearity is, the greater the amplitude of the wave is, which is demonstrated by the propagation of the sinusoidal wave and the distortion of its shape. The results obtained may be of practical interest, since the behavior of transverse phonons has a crucial importance in all the effects connected with the scattering of the conduction electron on the surface of the crystal $[20,21]$.

\section{Conflict of Interests}

The authors declare that there is no conflict of interests regarding the publication of this paper.

\section{Acknowledgments}

E. Pelinovsky thanks the support of the Volkswagen Stiftung and RFBR (14-05-00092).

\section{References}

[1] K. L. Ekinci and M. L. Roukes, "Nanoelectromechanical systems," Review of Scientific Instruments, vol. 76, no. 6, Article ID 061101, 2005.

[2] M. Stroscio and M. Dutta, Phonons in Nanostructures, Cambridge University Press, Cambridge, Mass, USA, 2001.

[3] M. Maldovan, "Sound and heat revolutions in phononics," Nature, vol. 503, pp. 209-217, 2013.

[4] A. Fasolino, J. H. Los, and M. I. Katsnelson, "Intrinsic ripples in graphene," Nature Materials, vol. 6, no. 11, pp. 858-861, 2007.

[5] J. C. Meyer, A. K. Geim, M. I. Katsnelson, K. S. Novoselov, T. J. Booth, and S. Roth, "The structure of suspended graphene sheets," Nature, vol. 446, no. 7131, pp. 60-63, 2007.
[6] X. Li, K. Maute, M. L. Dunn, and R. Yang, "Strain effects on the thermal conductivity of nanostructures," Physical Review B, vol. 81, no. 24, Article ID 245318, 2010.

[7] O. B. Gorbacheva and L. A. Ostrovsky, "Nonlinear vector waves in a mechanical model of a molecular chain," Physica D, vol. 8, no. 1-2, pp. 223-238, 1983.

[8] O. V. Rudenko and E. V. Solodov, "Strongly nonlinear shear perturbations in discrete and continuous cubic nonlinear systems," Acoustical Physics, vol. 57, no. 1, pp. 51-58, 2011.

[9] G. A. Maugin, Nonlinear Waves in Elastic Crystals, University Press, Oxford, UK, 1999.

[10] S. Takeno, S. V. Dmitriev, P. G. Kevrekidis, and A. R. Bishop, "Nonlinear lattices generated from harmonic lattices with geometric constraints," Physical Review B, vol. 71, no. 1, Article ID 014304, pp. 014304-014312, 2005.

[11] O. V. Rudenko and C. M. Hedbergb, "Strong and weak nonlinear dynamics: models, classification, examples," Acoustical Physics, vol. 59, no. 6, pp. 644-650, 2013.

[12] S. P. Nikitenkova and E. N. Pelinovsky, "Analysis of RudenkoSolodov equation in theory strongly nonlinear shear waves," Acoustical Physics, vol. 60, no. 3, pp. 240-242, 2014.

[13] V. E. Panin, V. E. Egorushkin, A. V. Panin, and D. D. Moiseenko, "On the nature of plastic strain localization in solids," Technical Physics, vol. 52, no. 8, pp. 1024-1030, 2007.

[14] S. Toyooka, R. Widiastuti, Q. Zhang, and H. Kato, "Dynamic observation of localized strain pulsation generated in the plastic deformation process by electronic speckle pattern interferometry," Japanese Journal of Applied Physics, vol. 40, no. 2, pp. 873876, 2001.

[15] O. V. Rudenko and S. I. Soluyan, Theoretical Foundations of Nonlinear Acoustics, Consultants Bureau, New York, NY, USA, 1977.

[16] S. N. Gurbatov, A. N. Malakhov, and A. I. Saichev, Nonlinear Random Waves and Turbulence in Nondispersive Media: Waves, Rays and Particles, Manchester University Press, 1991.

[17] O. V. Rudenko, "Nonlinear dynamics of quadratically cubic systems," Physics-Uspekhi, vol. 56, no. 7, pp. 683-690, 2013.

[18] E. Kartashova, E. Pelinovsky, and T. Talipova, "Fourier spectrum and shape evolution of an internal Riemann wave of moderate amplitude," Nonlinear Processes in Geophysics, vol. 20, no. 4, pp. 571-580, 2013.

[19] D. Pelinovsky, E. Pelinovsky, E. Kartashova, T. Talipova, and A. Giniyatullin, "Universal power law for the energy spectrum of breaking Riemann waves," JETP Letters, vol. 98, no. 4, pp. 237241, 2013

[20] A. C. Betz, F. Vialla, D. Brunel et al., "Hot electron cooling by acoustic phonons in graphene," Physical Review Letters, vol. 109, no. 5, Article ID 056805, 2012.

[21] H.-D. Wang, B.-Y. Cao, and Z.-Y. Guo, "Heat flow choking in carbon nanotubes," International Journal of Heat and Mass Transfer, vol. 53, no. 9-10, pp. 1796-1800, 2010. 


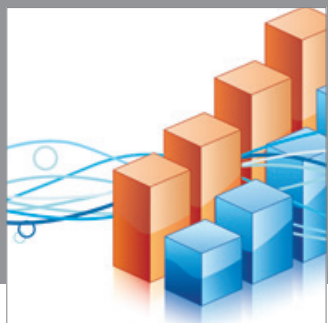

Advances in

Operations Research

mansans

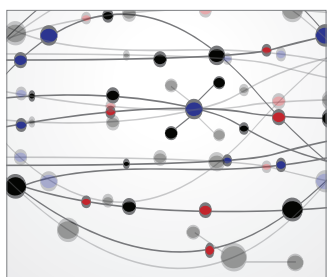

The Scientific World Journal
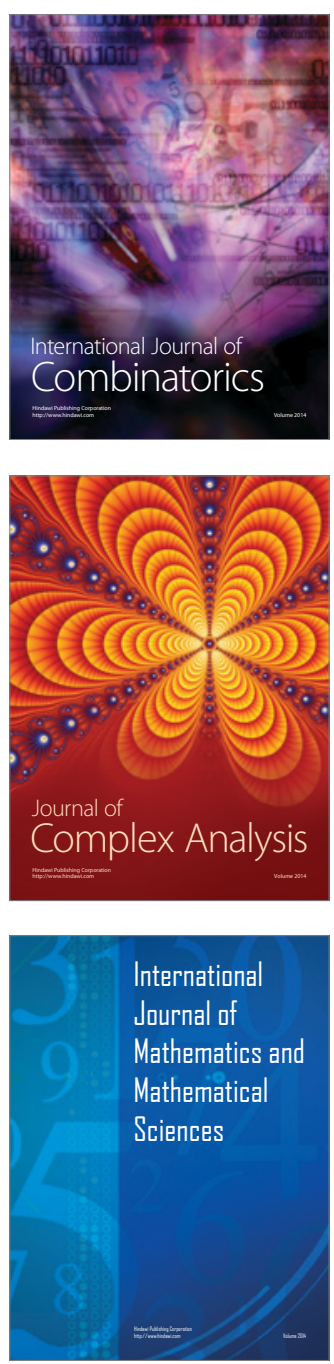
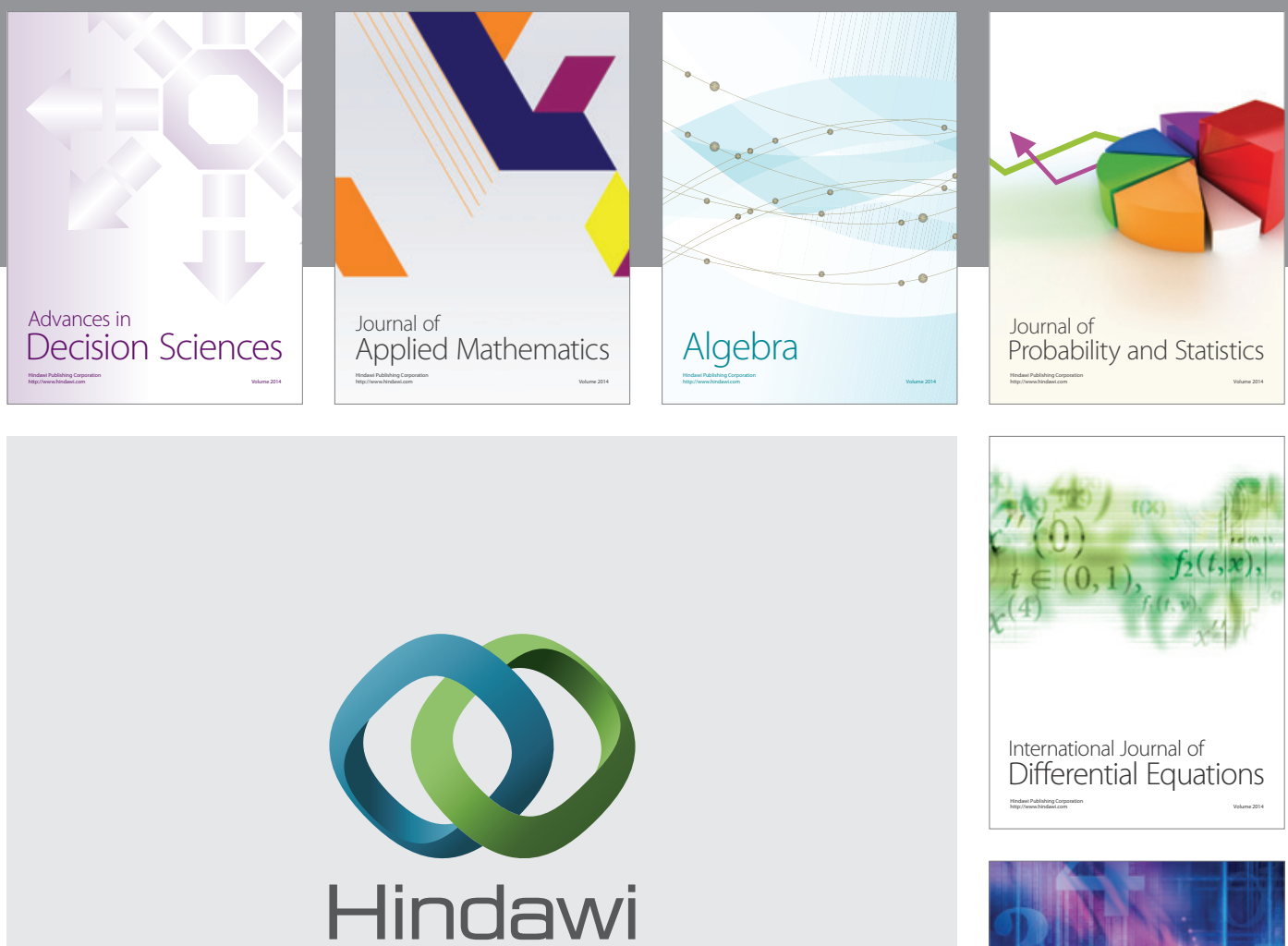

Submit your manuscripts at http://www.hindawi.com
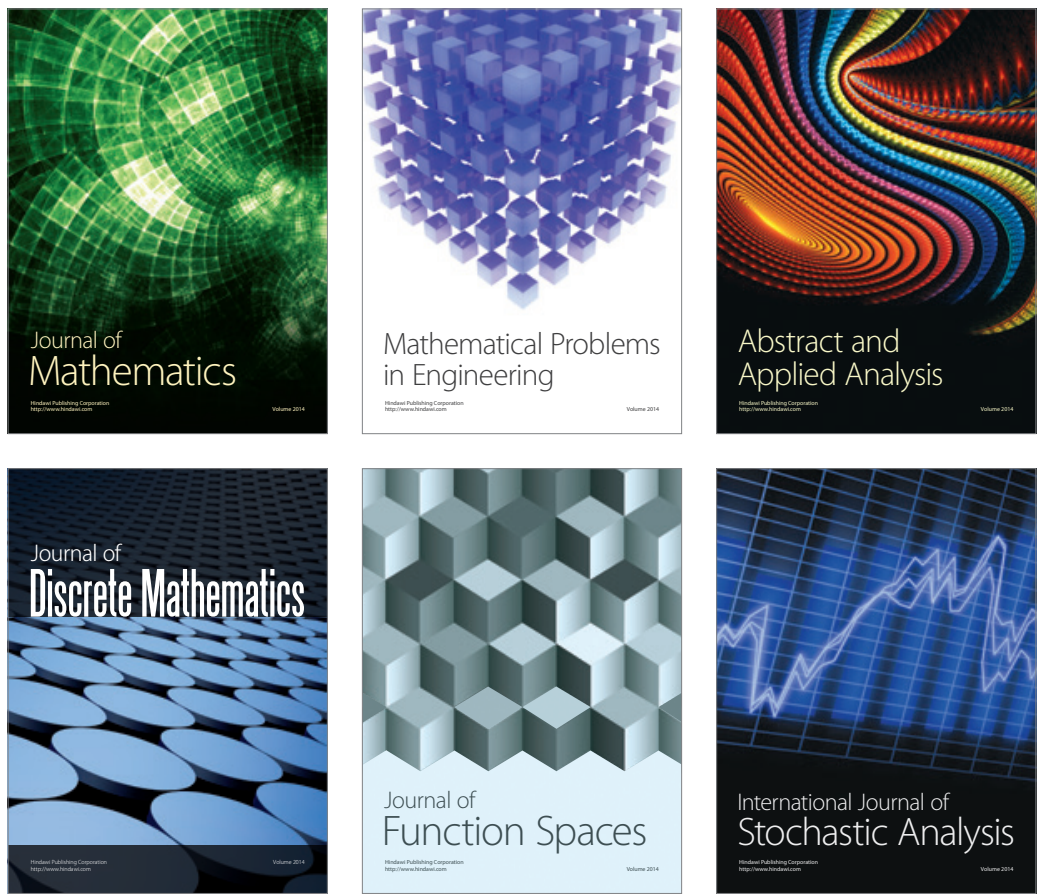

Journal of

Function Spaces

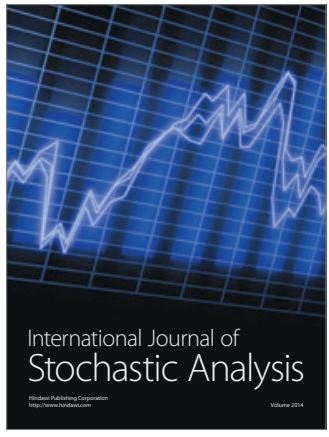

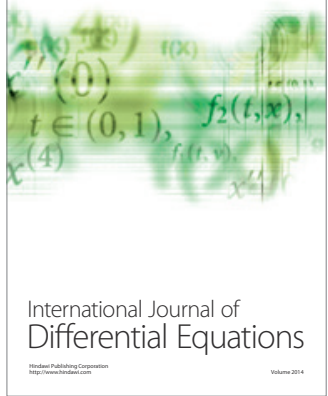
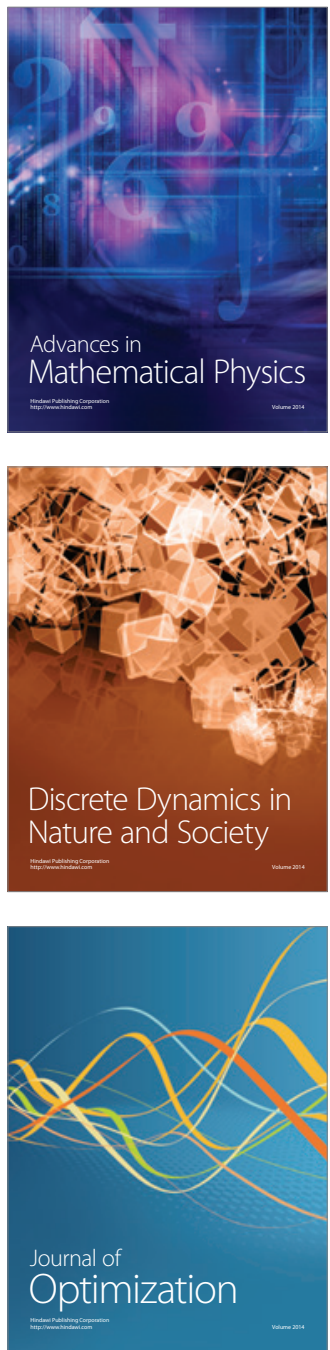